\title{
COUNTABLE PARACOMPACTNESS AND WEAK NORMALITY PROPERTIES
}

\author{
BY \\ JOHN MACK
}

In [4], Dowker proved that a normal space $X$ is countably paracompact if and only if its product with the closed unit interval is normal. In this paper, we prove an analogue of Dowker's theorem. Specifically, we define the term $\delta$-normal and then prove the following:

THEOREM 1. A topological space is countably paracompact if and only if its product with the closed unit interval is $\delta$-normal.

After proving this theorem, we obtain similar results for the topological spaces studied in [7] and [11]. Also, cogent examples are given and the relation this note bears to the work of others is discussed.

We shall follow the terminology of [5] except we shall assume separation properties for a space only when these assumptions are explicitly stated.

For an infinite cardinal $\mathfrak{m}$, a set $A$ in a topological space will be called a $G_{\mathfrak{m}}$-set (respectively, a regular $G_{\mathfrak{m}}$-set ) provided it is the intersection of at most $\mathfrak{m}$ open sets (respectively, at most $\mathfrak{m}$ closed sets whose interiors contain $A$ ). If $\mathfrak{m}=\boldsymbol{\aleph}_{0}$, we shall use the familiar terms $G_{\delta}$-set and regular $G_{\delta}$-set.

It is clear that the zero-set of any continuous real valued function is a regular $G_{\delta}$-set and that the intersection of no more than $m$ such zero-sets is a regular $G_{\mathrm{m}^{-}}$ set. In the remaining part of this paper, we shall use these facts without explicitly mentioning them.

Definition. For an infinite cardinal $\mathfrak{m}$, a topological space is $\mathfrak{m}$-normal if each pair of disjoint closed sets, one of which is a regular $G_{\mathfrak{m}}$-set, have disjoint neighborhoods. For $\mathfrak{m}=\boldsymbol{\aleph}_{0}$, we shall use the more suggestive term $\delta$-normal.

Note that a normal space is $m$-normal and that a regular space is normal if and only if it is $m$-normal for every infinite cardinal $\mathfrak{m}$. On the other hand, a compact $T_{1}$-space that is not Hausdorff is $m$-normal for every infinite cardinal but yet it fails to be normal.

Recall that a space is $\mathrm{m}$-paracompact if each open cover having cardinal less than or equal to $\mathfrak{m}$ has a locally finite open refinement. Characterizations of $\mathfrak{m}$ paracompact spaces may be found in [14] and [8].

THEOREM 2. Each m-paracompact space is $\mathfrak{m}$-normal.

Presented to the Society, January 25, 1970; received by the editors June 16, 1969.

Copyright (C) 1970, American Mathematical Society 
Proof. Suppose $X$ is m-paracompact and let $A$ and $B$ be disjoint closed sets such that $B$ is a regular $G_{\mathfrak{m}}$-set. Then there is a family $\mathscr{G}$, having cardinal less than or equal $\mathrm{m}$, consisting of open neighborhoods of $B$ such that $B$ is the intersection of $\{\bar{G}: G \in \mathscr{G}\}$. Let $\Gamma$ be the set of all finite nonempty subfamilies of $\mathscr{G}$. For $\alpha \in \Gamma$, define $G_{\alpha}$ to be the intersection of $\{G: G \in \alpha\}$. Then $B \subset G_{\alpha}$ and $B=$ $\cap \bar{G}_{\alpha}$. The family of sets $X \backslash A \cap \bar{G}_{\alpha}$ is a directed open cover of $X$. By Theorem 5 in [8], there exists a locally finite open cover $\left\{V_{\alpha}\right\}$ such that $\bar{V}_{\alpha} \cap A \cap \bar{G}_{\alpha}=\varnothing$ for all $\alpha$ in $\Gamma$. Now let $U$ be the union of all sets $V_{\alpha} \mid \bar{G}_{\alpha}$ and $V$ be the union of the sets $G_{\alpha} \backslash \cup\left\{\bar{V}_{\beta}: \beta\right.$ is not a subset of $\left.\alpha\right\}$. Clearly, $U$ is open, contains $A$ and is disjoint from $V$. Since $\left\{V_{\alpha}\right\}$ is locally finite it follows that $V$ is open. For $x \in B$, let $\gamma=$ $\bigcup\left\{\beta: x \in \bar{V}_{\beta}\right\}$. Since the cover $\left\{V_{\alpha}\right\}$ is locally finite, $\gamma \in \Gamma$ and $x \notin \bigcup\left\{\bar{V}_{\beta}: \beta \nsubseteq \gamma\right\}$; whence $x \in V$. Therefore $B \subset V$ and the proof is complete.

For the important special case where $\mathfrak{m}=\boldsymbol{\aleph}_{0}$, we have:

THEOREM 3. Each countably paracompact space is $\delta$-normal.

The local weight of a topological space is the least cardinal $m$ such that each point has a neighborhood base consisting of at most $m$ elements.

THEOREM 4. (i) A Hausdorff $\mathrm{m}$-normal space having local weight $\leqq \mathfrak{m}$, is regular.

(ii) A Hausdorff $\mathfrak{m}$-normal space having cardinal $\leqq \mathfrak{m}$, is regular.

Proof. Under the hypotheses in each case, a singleton is a regular $G_{\mathfrak{m}}$-set.

For emphasis, we state the following special case:

THEOREM 5. If a $\delta$-normal Hausdorff space is either countable or satisfies the first axiom of countability, then it is regular.

COROLlaRy 6 (Aull [1]). Each countably paracompact, first countable, Hausdorff space is regular.

EXAMPLE. For each infinite cardinal $\mathfrak{m}$, there is an $\mathfrak{m}$-normal, Hausdorff space which is not regular. Given $\mathfrak{m}$, let $w_{\alpha}$ be the least ordinal having cardinal greater than $m$. Denote by $W^{*}$ the set of ordinals less than or equal to $w_{\alpha}$ and by $W$, the set $W^{*} \mid\left\{w_{\alpha}\right\}$. In $W^{*} \times W^{*} \mid\left\{\left(w_{\alpha}, w_{\alpha}\right)\right\}$, identify all points of $W \times\left\{w_{\alpha}\right\}$. The quotient space $X$ is Hausdorff but not regular (the images in $X$ of the upper edge and the diagonal are not separated by disjoint open sets). Nonetheless, $X$ is m-compact; hence it is $\mathfrak{m}$-paracompact and $\mathfrak{m}$-normal.

Throughout this paper $I$ will denote the closed unit interval. In the proof of Theorem 1, we shall use the following lemma.

LEMMA 7. For any topological space $X$, the following are equivalent:

(a) $X$ is countably paracompact.

(b) If $g$ is a strictly positive lower semicontinuous function on $X$, then there exist real valued functions $l$ and $u$ with $l$ lower semicontinuous and $u$ upper semicontinuous such that $0<l(x) \leqq u(x) \leqq g(x)$ for all $x \in X$. 
(c) If $A$ is a closed subset of $X \times I$ and $K$ is closed in I such that $A$ and $X \times K$ are disjoint, then $A$ and $X \times K$ have disjoint neighborhoods.

Proof. The equivalence of (a) and (c) is due to Tamano (Theorem 3.9 in [16]) while the equivalence of (a) and (b) is an easy consequence of Theorem 10 in [7].

Proof of Theorem 1. If $X$ is countably paracompact, then $X \times I$ is countably paracompact (Theorem 1 in [4]). By Theorem 3 above $X \times I$ is $\delta$-normal. Conversely, suppose $K$ is closed in $I$. Since $I$ is metrizable, $K$ is a regular $G_{\delta}$-set. Therefore $X \times K$ is a regular $G_{\delta}$-set in $X \times I$. In view of Lemma 7, the $\delta$-normality of $X \times I$ will imply that $X$ is countably paracompact.

THEOREM 8. A closed continuous image of an m-normal space is m-normal.

Proof. Observe that a continuous inverse image of a regular $G_{\mathrm{m}}$-set is regular $G_{\mathfrak{m}}$. Using this fact, the standard proof that a closed continuous image of a normal space is normal, becomes applicable here.

REMARK. In general, it is not true that preimages of $\mathfrak{m}$-normal spaces are $\mathfrak{m}$ normal even for perfect maps (i.e., continuous closed maps for which the preimages of compact sets are compact). Note that in view of Theorem 1 and the fact that a space $X$ is always the perfect image of $X \times I$, it follows that if every perfect preimage of $X$ is $\delta$-normal, then $X$ is countably paracompact.

Many of the standard examples of nonnormal spaces, also, fail to be $\delta$-normal. Here we give a partial list of such examples. (i) The space $S \times S$ where $S$ is the reals with the half-open interval topology [15]. (ii) The space $X \times Y$ constructed by Michael [12]. (iii) The space $R^{R}$ where $R$ is the space of reals. (iv) The spaces constructed in problems $3 \mathrm{~K}, 5 \mathrm{I}, 6 \mathrm{P}, 6 \mathrm{Q}$ of [5]. We shall use the space $S \times S$ to illustrate a technique that can be used to verify that these spaces are not $\delta$-normal. In $S \times S$ let $A$ be the set of points $(x,-x)$ where $x$ is rational and $B$ be the set of such points for irrational $x$. Then $A$ is closed and $B$ is a regular $G_{\delta}$-set while these sets do not have disjoint neighborhoods. To show this, one can exploit the fact that the irrationals are not an $F_{\sigma}$-set in the reals (cf. [12]).

Definition. A space will be called $\delta$-normally separated if each closed set and each zero set disjoint from it are completely separated. A space will be termed weakly $\delta$-normally separated if each regular closed set (i.e., the closure of an open set) and zero-set disjoint from it are completely separated.

REMARK. The properties of being $\delta$-normal and $\delta$-normally separated are, unfortunately, not comparable for arbitrary topological spaces. In a space where every regular $G_{\delta}$-set is a zero-set, $\delta$-normal separation implies $\delta$-normality, but not conversely (see the example at the end of this paper). On the other hand, Hewitt's example [6] of an infinite regular Hausdorff space on which each continuous real-valued function is constant, is a $\delta$-normally separated space which is not $\delta$-normal (cf. Remark following Theorem 13). The author does not know whether among completely regular spaces, $\delta$-normal separation implies $\delta$ normality. 
Clearly each normal space is $\delta$-normally separated. Likewise, $\delta$-normal separation implies weak $\delta$-normal separation and the converse is true for $\delta$-normal spaces.

The concept of $\delta$-normal separation is not a new one. P. Zenor introduced this idea in [17] and used the term Property $Z$.

The $\delta$-normal separation of a space $X$ can be characterized in terms of properties of the ring $C(X)$ of a real-valued continuous function on $X$.

THEOREM 9. A topological space $X$ is $\delta$-normally separated if and only if for each $f \in C(X)$ and each closed set $A$ on which $f$ is strictly positive, there exists a unit $u$ of the ring $C(X)$ such that $f u$ is identically one on $A$.

Proof. Assume $X$ is $\delta$-normally separated and that $f$ and $A$ have the given properties. Then there exists a nonnegative element $h$ of $C(X)$ which vanishes on $A$ and assumes the value 1 everywhere on the zero-set of $f$. Then the ring inverse of $|f|+h$ is the desired unit. The converse is obvious.

We shall now proceed to state and prove the analogue of Theorem 1 for the $\delta$-normal separation and weak $\delta$-normal separation properties. To achieve this, we need to recall the definitions of $c b$-spaces and weak $c b$-spaces. A space $X$ is a $c b$-space (respectively, weak $c b$-space) provided every locally bounded real valued function on $X$ (respectively, every locally bounded lower semicontinuous function on $X$ ) is bounded above by a continuous function. Information concerning $c b$ spaces and weak $c b$-spaces may be found iil [7] and [11], respectively. In comparing Theorem 1 with Theorem 11 below, it is useful to remember that a space is $c b$ if and only if it is weak $c b$ and countably paracompact.

LEMMA 10. (a) Each cb-space is $\delta$-normally separated.

(b) Each weak cb-space is weakly $\delta$-normally separated.

Proof. We shall prove (a) and make parenthetical comments to indicate the proof of (b). Let $A$ be closed and $Z$ be a zero-set disjoint from $A$. Given a nonnegative function $h$ in $C(X)$ such that $Z$ is the zero-set of $h$, define $g(x)=1+h(x)$ for $x$ not in $A$ and $g(x)=h(x)$ for $x$ belonging to $A$. Then $g$ is lower semicontinuous (normal lower semicontinuous if $A$ is regular closed). Clearly $g$ is strictly positive. By Theorem 1 in [7] (Theorem 3.1 in [11] for (b)) there is a strictly positive real valued continuous function $f$ such that $f \leqq g$. Then the function $h / f$ completely separates $A$ and $Z$.

THEOREM 11. Let $X$ be a topological space. Then

(a) $X$ is a cb-space if and only if $X \times I$ is $\delta$-normally separated.

(b) $X$ is a weak cb-space if and only if $X \times I$ is weakly $\delta$-normally separated.

Proof. The necessity follows from Lemma 10 above; the sufficiency from Corollary 12 and Theorem 13 in [9].

Remark, In both Theorems 1 and $11, I$ may be replaced by any infinite compact 
metric space. Also, note that Theorem 10 in [9] implies that a variation of (a) in the above theorem is valid when $I$ is replaced by an infinite product of intervals.

COROLlaRY 12. (a) Each countably compact space is both $\delta$-normal and $\delta$ normally separated.

(b) A completely regular, pseudocompact space is weakly $\delta$-normally separated.

Proof. Since countably compact spaces are $c b$ (Corollary 3 in [7]) and completely regular pseudocompact spaces are weak $c b$ (Corollary 3.8 in [11]), this theorem follows immediately from Lemma 10.

It is well known that a normal pseudocompact Hausdorff space is countably compact. In [17], Zenor shows that normality may be replaced by $\delta$-normal separation. Here we show the condition can be further weakened to $\delta$-normality.

THEOREM 13. A completely regular space is countably compact if and only if it is $\delta$-normal and pseudocompact.

Proof. By Corollary 12 above, a pseudocompact $\delta$-normal space is also $\delta$ normally separated. This theorem now follows from Zenor's result (Theorem 3 in [17]).

REMARK. In Theorem 13, it is essential that the space be completely regular; for there exist regular, countably paracompact, Hausdorff spaces, that are not countably compact, on which every real valued function is constant [10]. Such a space can be obtained by altering slightly the construction used by Hewitt in [6].

For a completely regular space $X$, let $v X$ denote the Hewitt realcompactification. In [5, p. 120], it is noted that the normality of $X$ and of $v X$ are independent of each other. The same is true for $\delta$-normality and $\delta$-normal separation. To see this, first, let $X$ be a completely regular pseudocompact space that is not countably compact (the Tychonoff plank will do nicely). Then $v X$ is compact and hence is both $\delta$-normal and $\delta$-normally separated, but $X$ has neither of these properties. On the other hand, let $P$ be the product $R^{R}$ of $\mathrm{c}(\mathrm{c}=$ card $R)$ copies of the reals $R$ and let $X$ be an associated $\Sigma$-product. Then $X$ is normal, and $v X=P$ (see [3]) but $P$ is not countably paracompact. Whence it follows from Theorem 1 that $P$ is not $\delta$-normal and from Theorem 11 that $P$ is not $\delta$-normally separated.

The situation for weak $\delta$-normal separation is entirely different as Theorems 14 and 17 below will show.

THEOREM 14. If a completely regular Hausdorff space $X$ is weakly $\delta$-normally separated then $v X$ is as well.

Proof. If $A$ is regular closed in $v X$ and $Z$ is a zero-set in $v X$, then $A \cap X$ is regular closed in $X$ and $Z \cap X$ is a zero-set in $X$. Moreover, $A$ and $Z$ are the closures in $v X$ of $A \cap X$ and $Z \cap X$ respectively (for the latter see 8.8(b) in [5]). If $f$ is a continuous real valued function on $X$ which completely separates $A \cap X$ and $Z \cap X$, then its extension to $v X$ clearly separates $A$ from $Z$. 
COROLlaRY 15. Any product of complete separable metric spaces is weakly $\delta$-normally separated.

Proof. In [3], it is proved that any such product is $v X$ for some normal space $X$.

In order to obtain a partial converse of Theorem 14, we prove the following lemma which seems to be of independent interest. A point $x$ of a space $X$ is a $q$-point [13] if it has a sequence $\left\{U_{n}\right\}$ of neighborhoods such that if $\left\{x_{n}\right\}$ is a sequence of distinct points with $x_{n} \in U_{n}$, then this sequence has an accumulation point.

LEMMA 16. If every point of $v X \backslash X$ is a q-point of $v X$, then every pair of disjoint sets $A, Z$ where $A$ is regular closed in $X$ and $Z$ is a zero-set in $X$, have disjoint closures in $v X$.

Proof. Suppose on the contrary that $p$ belongs to the closure of both $A$ and $Z$ and let $\left\{U_{n}\right\}$ be a sequence of open neighborhoods of $p$ given by the definition of $q$-points. Let $G$ denote the interior of $A$ in $X$ and $f \in C(X)$ be a function whose zero-set is $Z$. By our assumption $p$ belongs to the closure of $G \cap\{x:|f(x)|<1 / n\}$ (call this set $H_{n}$ ) for each positive integer $n$. Whence $U_{n} \cap H_{n}$ is nonempty for each $n$. Pick $x_{n}$ from this set. Clearly, we may assume that the $x_{n}$ are distinct. Since $A$ and $Z$ are disjoint, it follows that $\left\{H_{n}\right\}$ is locally finite. Thus the sequence $\left\{x_{n}\right\}$ has no accumulation point. But this is impossible since $p$ is a $q$-point.

THEOREM 17. If $v X$ is locally compact (more generally, if each point in $v X \backslash X$ has a compact neighborhood in $v X)$, then $X$ is weakly $\delta$-normally separated if and only if $v X$ has the same property.

A converse for Theorem 14 is not possible, without some sort of restriction on $v X$. This is shown in the example below.

It is natural to ask what relation the above results bear to the well known unanswered question [4, p. 221]: Must the product of a normal Hausdorff space with the closed unit interval be normal? In this regard, first, observe that if $X$ is normal, then $X \times I$ is normal provided it is $\delta$-normal. This fact suggests the following question: If $X$ is a regular, $\delta$-normal space, must $X \times I$ be $\delta$-normal ? Except for noting that without the assumption that the space is regular, the answer to this question is negative (see p. 221 in [4]), the author has not obtained any significant clues concerning the answer to this question. On the other hand, the answer to the corresponding question for $\delta$-normal separation is negative. This is the substance of the following example.

EXAMPLE. Let $X$ and $X^{*}$ be the spaces constructed on pp. 240, 241 of [11]. There it is pointed out that $X$ is locally compact, countably paracompact but not a $c b$-space while $X^{*}$ is $\sigma$-compact but not locally compact and that $X^{*}=v X$. It is a simple matter (using Theorem 9 and the special relation that $X$ bears to $X^{*}$ ) to show that $X$ is also $\delta$-normally separated. Since $X$ is not a $c b$-space, it follows from Theorem 11 that $X \times I$ is not $\delta$-normally separated (or even weakly $\delta$ normally separated). It is, however, $\delta$-normal. 
Also, in view of Theorem 2.8 in [2], note that $v(X \times I)=v X \times I$. Now since $v X \times I=X^{*} \times I$ is Lindelöf and regular, it is normal. Nonetheless $X \times I$ fails to be weakly $\delta$-normally separated. This shows that the restriction on $v X$ in Theorem 17 cannot be entirely suppressed.

In [14], Morita obtained the following generalization of Dowker's theorem [4]: A space $X$ is $\mathrm{m}$-paracompact and normal if and only if $X \times I^{\mathfrak{m}}$ is normal. In view of Morita's result, it is natural to ask: What condition on $X$ is necessary and sufficient for $X \times I^{\mathrm{m}}$ to be $\mathrm{m}$-normal? Theorem 2 implies that $\mathfrak{m}$-paracompactness of $X$ is a sufficient condition; however the author has been unable to determine whether $m$-paracompactness is also necessary. The chief stumbling block is the lack of a characterization of $\mathrm{m}$-paracompactness similar to that for countable paracompactness given by Tamano (Lemma 7 above).

In contrast to the obstacles encountered in attempting to obtain an analogue of Theorem 1 for uncountable cardinals, Theorem 11 (as pointed out in the Remark following that theorem) can be extended by merely giving an appropriate meaning to the term $m$-normal separation. Specifically, define a space to be $\mathfrak{m}$-normally separated provided the intersection of any family consisting of at most $\mathfrak{m}$ zero-sets is completely separated from any closed set disjoint from it. Then for any space $X$, $X \times I^{\mathfrak{m}}$ is $\mathfrak{m}$-normally separated if and only if $X$ is an $H(\mathfrak{m})$-space in the sense of [9].

\section{REFERENCES}

1. C. E. Aull, A note on countably paracompact spaces and metrization, Proc. Amer. Math. Soc. 16 (1965), 1316-1317. MR 32 \#3039.

2. W. W. Comfort and S. Negrepontis, Extending continuous functions on $X \times Y$ to subsets of $\beta X \times \beta Y$, Fund. Math. 59 (1966), 1-12. MR 34 \#782.

3. H. H. Corson, Normality in subsets of product spaces, Amer. J. Math. 81 (1959), 785-796. MR 21 \#5947.

4. C. H. Dowker, On countably paracompact spaces, Canad. J. Math. 3 (1951), 219-224. MR 13, 264.

5. L. Gillman and M. Jerison, Rings of continuous functions, The University Series in Higher Math., Van Nostrand, Princeton, N. J., 1960. MR 22 \#6994.

6. E. Hewitt, On two problems of Urysohn, Ann. of Math. (2) 47 (1946), 503-509. MR 8, 165.

7. John Mack, On a class of countably paracompact spaces, Proc. Amer. Math. Soc. 16 (1965), 467-472. MR 31 \#1651.

8. - Directed covers and paracompact spaces, Canad. J. Math. 19 (1967), 649-654. MR 35 \#2263.

9. - Product spaces and paracompactness, J. London Math. Soc. (2) 1 (1969), 90-94.

10. - Countably paracompact spaces on which every real-valued continuous function is constant, Proc. Amer. Math. Soc. (to appear).

11. John Mack and D. G. Johnson, The Dedekind completion of $C(\mathscr{X})$, Pacific J. Math. 20 (1967), 231-243. MR 35 \#2150.

12. E. Michael, The product of a normal space and a metric space need not be normal, Bull. Amer. Math. Soc. 69 (1963), 375-376. MR 27 \#2956. 
13. E. Michael, A note on closed maps and compact sets, Israel J. Math. 2 (1964), 173176. MR 31 \#1659.

14. K. Morita, Paracompactness and product spaces, Fund Math. 50 (1961/62), 223-236. MR 24 \#A2365.

15. R. H. Sorgenfrey, On the topological product of paracompact spaces, Bull. Amer. Math. Soc. 53 (1947), 631-632. MR 8, 594.

16. H. Tamano, On compactifications, J. Math. Kyoto Univ. 1 (1961/62), 161-193. MR 25 \#5489.

17. P. Zenor, A note on Z-mapping and WZ-mappings, Proc. Amer. Math. Soc. 23 (1969), 273-275.

UNIVERSITY OF KENTUCKY,

LEXINGTON, KENTUCKY 